\title{
Investigating the Firing Protocol of Athenian Pottery Production: A Raman and Hi-Resolution TEM Study
}

\author{
Ilaria Cianchetta ${ }^{2}$, Karen Trentelman ${ }^{2}$, Marc Walton $^{3}$, Apurva Mehta ${ }^{4}$, Brendan Foran ${ }^{1}$ \\ ${ }^{1}$ The Aerospace Corporation, El Segundo, California, \\ ${ }^{2}$ Getty Conservation Institute, Los Angeles, California \\ ${ }^{3}$ Northwestern University/Art Institute of Chicago, Chicago, Illinois \\ ${ }^{4}$ Stanford Synchrotron Radiation Lightsource, Stanford University, Palo Alto, California
}

The decorated ceramics produced in ancient Athens from the 6th through the 4th centuries BCE, also known as Attic pottery, are considered a benchmark technological achievement of the pre-industrial world. The iconic black-figure vessels (black figures painted on red reserve), and the later, more refined, red-figure vessels (figures left in reserve on a black background), demonstrate a sophisticated control of particle morphology, porosity and firing conditions. Both techniques achieved the production of smooth and glossy black surfaces layers - called black gloss - by alternating the high-temperature kiln between oxidative and reductive environments during firing. Areas painted with a refined slip turned from red (due to the presence of $\mathrm{Fe} 3+$ compounds: hematite, $\alpha-\mathrm{Fe}_{2} \mathrm{O}_{3}$ and maghemite, $\gamma-\mathrm{Fe}_{2} \mathrm{O}_{3}$ ) to black (Fe2+ iron compounds: magnetite, $\mathrm{Fe}_{3} \mathrm{O}_{4}$, hercynite, $\mathrm{FeAl}_{2} \mathrm{O}_{4}$ ) depending on the atmospheric condition of each stage. Although a basic understanding of their production has been developed, details regarding the painting and firing schemes employed are still not completely understood ${ }^{[1]}$.

As part of an ongoing study ${ }^{[2,3]}$, the work presented here examines the technological foundations of Athenian pottery production through the replication of the firing technology. Thirty-six replicate samples were painted with refined illitic clay and fired using a three-stage (oxidation-reductionoxidation) firing protocol, systematically varying the temperature, oxygen fugacity, humidity and duration of each stage. The resulting gloss samples ranged in color from bright red to deep black, as well as "misfired" samples in which both red and black gloss is formed, all similar in appearance to examples found on ancient Athenian pottery. The samples were characterized by Raman Spectroscopy to determine the relative amount of magnetite (black) and hematite (red) present in the gloss, and High Resolution Transmission Electron Microscopy was used to characterize the morphology of the gloss, and assess the degree of vitrification.

Results indicate that the temperature of each of the oxidative steps - the first and third stages - are most important for controlling the color, the mineralogical phases and the density of the glosses produced. The re-oxidation in the third (oxidative) step is dependent on both the porosity of the slip (which is directly related to the amount of glassy phase formed in the first firing step) and oxygen diffusion through the slip. Based on our preliminary studies, we believe only few time/temperature combinations for each of the three stages lead to the formation of a black slip over a red body, as found in ancient Athenian pottery. While the formation of a liquid phase is crucial during the first stage, this condition would be detrimental during the last stage since oxygen diffusion through melted silica would re-oxidize the slip back to its original red color $\left(\mathrm{Fe}^{3+}\right.$ compounds) producing a dense red slip. From the results of these replication experiments, the firing conditions of ancient Athenian black and red glosses may be inferred. 


\section{References:}

[1] J.V. Noble "The technique of Attic vase painting" American Journal of Archaeology 64, (1960) 307313

[2]M. Walton et al "Characterization of coral red slips on Greek attic pottery" 2008, Archaeometry 51, 3 (2009) 383-396.

[3] M. Walton et al, "Material Evidence for Multiple Firings of Ancient Athenian Red-Figure Pottery" Journal of American Ceramic Society 96, 7, (2013) 2031-2035.

[4] This material is based upon work supported by the National Science Foundation under Grant No. 1041808. The authors also gratefully acknowledge Jeffrey Maish and David Saunders of the J. Paul Getty Museum for their contributions to this work.
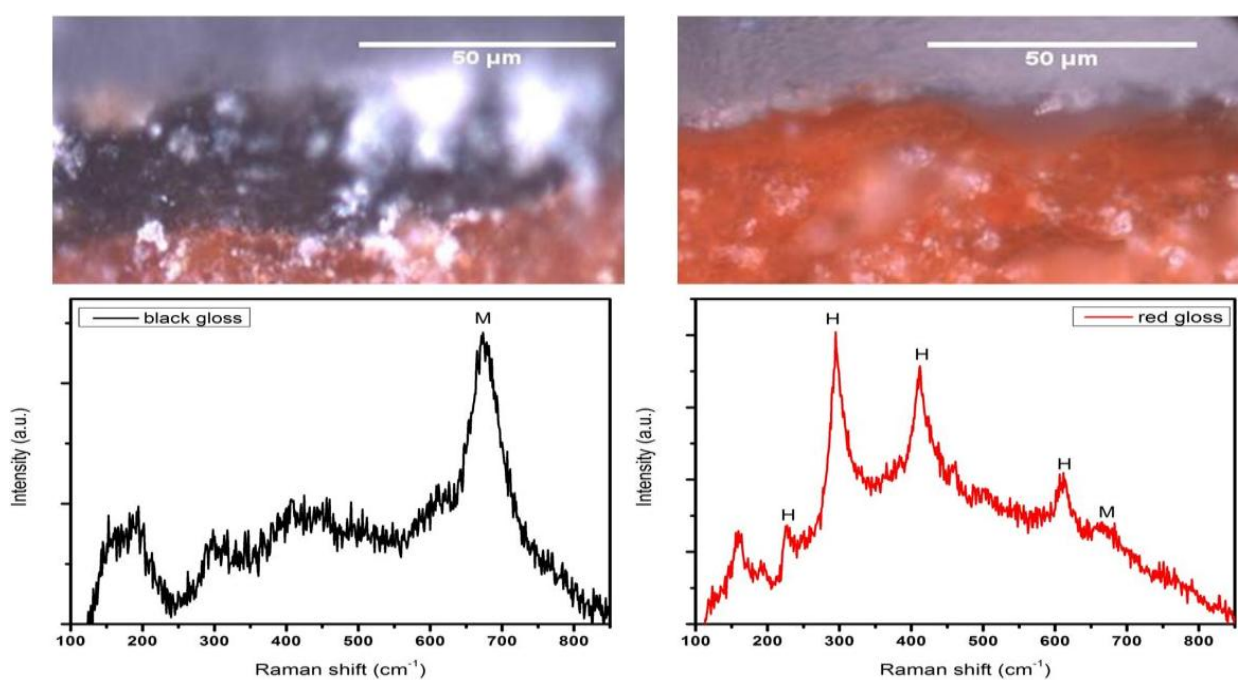

Fig. 1: Cross-section and Raman spectrum of (left) black gloss fired at $950^{\circ} \mathrm{C}$ (ox) $-850^{\circ} \mathrm{C}$ (red) $-900^{\circ} \mathrm{C}$ (ox); (right) red gloss fired at $700^{\circ} \mathrm{C}$ (ox) $-850^{\circ} \mathrm{C}$ (red) $-900^{\circ} \mathrm{C}$ (ox). $\mathrm{M}=$ magnetite; $\mathrm{H}=$ hematite.
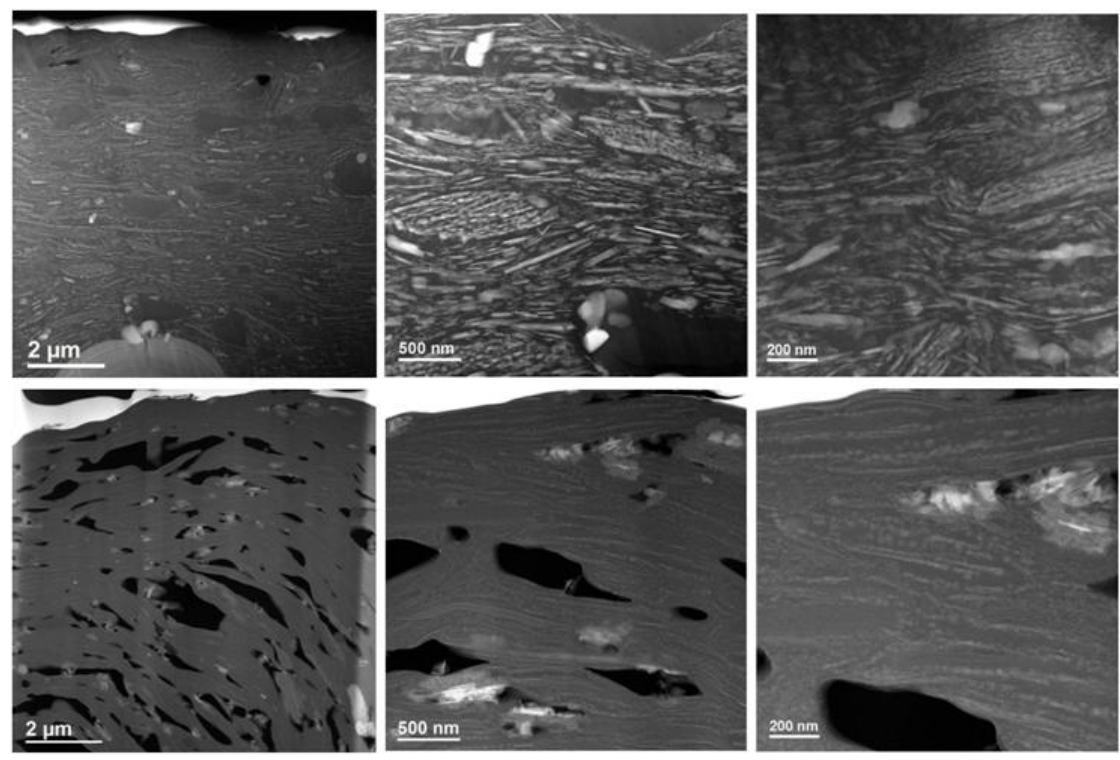

Figure 2: Hi-Res TEM images of (top) replicate of black relief line; fired at $950^{\circ} \mathrm{C}$ (ox) $-850^{\circ} \mathrm{C}$ (red) $900^{\circ} \mathrm{C}$ (ox), and (bottom) replicate red gloss; fired at $700^{\circ} \mathrm{C}(\mathrm{ox})-850^{\circ} \mathrm{C}$ (red) $-900^{\circ} \mathrm{C}$ (ox). 